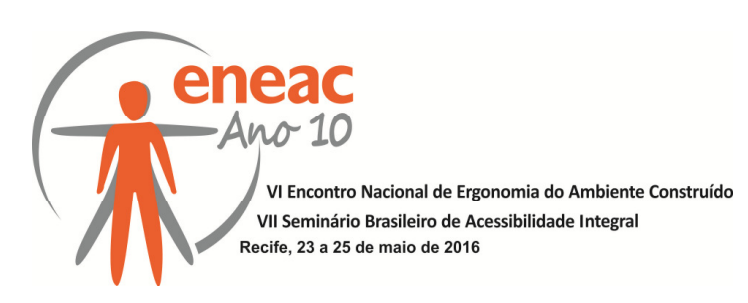

\title{
AVALIAÇÃO DAS CONDIÇÕES DE ACESSIBILIDADE ESPACIAL E PROPOSTA DE INTERVÉNÇÃO EM EDIFICAÇÃO DA UFPEL/RS
}

\author{
CANTARELLI, Rafaela (1); \\ POUEY, Maria Tereza (2); \\ ANDRADE, Isabela Fernandes (3) \\ (1) Universidade Federal de Pelotas, Graduada em Engenharia Civil \\ e-mail: rafa_cantarelli@yahoo.com.br \\ (2) Universidade Federal de Pelotas, Doutora em Engenharia \\ e-mail: mtpouey@brturbo.com.br \\ (3) Universidade Federal de Pelotas, Mestre em Arquitetura e Urbanismo \\ e-mail: acessiarq@gmail.com
}

\section{RESUMO (11 PTS, NEGRITO)}

\begin{abstract}
Apesar de garantido na legislação brasileira, o acesso à educação para todas as pessoas não é atendido na prática, pois, entre outros problemas, muitas edificações de uso educacional e público ainda não oferecem condições adequadas para receber indivíduos com algum tipo de limitação. Sendo assim, o trabalho teve como objetivos avaliar as condições de acessibilidade espacial e elaborar proposta de intervenção para o prédio do Departamento de Fisiologia e Farmacologia I da UFPel/RS. A edificação abrange diversas atividades acadêmicas, o que justifica sua escolha. Os métodos adotados foram visita exploratória e passeios acompanhados Os resultados deste estudo foram tratados de acordo com os quatro componentes de acessibilidade espacial: comunicação, deslocamento, orientação espacial e uso.
\end{abstract}

Palavras chave: Acessibilidade espacial, Edifício público, Universidade.

\section{ABSTRACT (11 PTS, NEGRITO)}

Although guaranteed by Brazilian law, access to education for all people is not met in practice because, among other problems, many buildings of educational and public use does not offer suitable conditions for individuals to receive some kind of limitation. Thus, the study aimed to evaluate the conditions of spatial accessibility and develop intervention proposal for the building of the Departamento de Fisiologia e Farmacologia I of UFPel/RS. The building includes various academic activities which justifies their choice. The methods adopted were exploratory visit and accompanied walks. The results of this study were treated according to the four components of spatial accessibility: communication, displacement, spatial orientation and use.

Keywords: Spatial Accessibility, Public Building, University. 


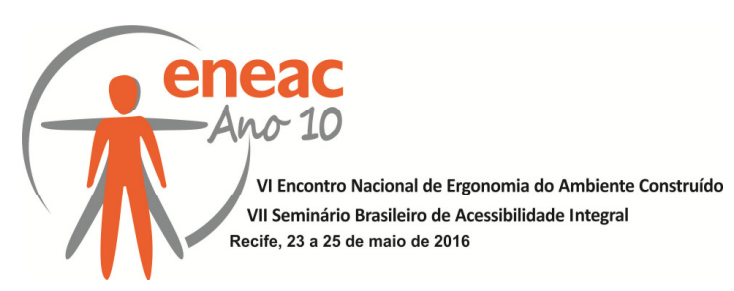

\section{INTRODUÇÃO (11 PTS, NEGRITO)}

A igualdade e a não discriminação entre as pessoas, no Brasil, são direitos garantidos pela Constituição da República Federativa criada em 1988 (BRASIL, 1988). Corroborando com tal legislação, o Ministério da Educação (MEC) instituiu, em 2003, a portaria № 3.284 assegurando os direitos das pessoas com deficiência no âmbito escolar, na qual estabelece requisitos de acessibilidade a serem incluídos nos instrumentos de avaliação para fins de autorização, reconhecimento, credenciamento e renovação de cursos de ensino superiores (BRASIL, 2003).

Embora seja garantido legalmente, o acesso à educação, na prática, ainda não é realidade para todas as pessoas, principalmente para aquelas com algum tipo de limitação, como mobilidade reduzida ou deficiências. Este cenário se deve ao fato de que a maioria dos edifícios públicos existentes no Brasil ainda não estão aptos a receberem todos os tipos de pessoas, o que segundo Dischinger et al. (2012), está relacionado à complexidade e a falta de embasamento sobre o tema na formação dos profissionais responsáveis pelas intervenções nas edificações visando torná-las acessíveis espacialmente.

Com o intuito de contribuir na transformação deste cenário, o presente trabalho teve como objetivos avaliar as condições de acessibilidade espacial e elaborar proposta de intervenção para o prédio do Departamento de Fisiologia e Farmacologia I da Universidade Federal de Pelotas (UFPel). A escolha do objeto de estudo se deu por envolver diferentes tipos de atividades acadêmicas, desde salas de aula até biblioteca, o que indica um elevado número de pessoas transitando pelas dependências desta edificação diariamente.

Neste artigo, é apresentada parte do conteúdo final elaborado no Trabalho de Graduação do Curso de Engenharia Civil da UFPel. Inicialmente, são expostos os problemas identificados na edificação analisada a partir de diferentes pontos de vista - do técnico, do usuário em cadeira de rodas e do usuário cego. E, por fim, é apresentada a proposta de adaptação elaborada para solucionar os problemas relacionados à acessibilidade espacial encontrados no edifício em questão. Sendo assim, a pesquisa trata-se de um estudo de caso, uma vez que busca retratar a realidade do objeto de estudo.

Espera-se, com este trabalho, contribuir com a adaptação de um dos prédios da UFPel às condições exigidas em legislação, além de servir como referência na continuidade deste processo.

\section{FUNDAMENTAÇÃO TEÓRICA}

Para melhor compreensão da questão de acessibilidade espacial é fundamental que se tenha o conhecimento do significado de termos como deficiência e restrição e da relação existente entre eles.

\subsection{ACESSIBILIDADE}

A NBR 9050/2004 define acessibilidade como sendo a "possibilidade e condição de alcance, percepção e entendimento para a utilização com segurança e autonomia de edificações, espaço, mobiliário, equipamento urbano e elementos." (ABNT, 2004, p.2). Tratada de uma forma ampla, a acessibilidade está ligada a todos os fatores que influenciam na realização das atividades, incluindo não somente os aspectos sociais, políticos, econômicos e culturais (analfabetismo, preço do transporte público, idiomas diferente, etc.), como também os físicoespaciais (deslocamento, distância e outros) (DISCHINGER, 2005). Neste estudo foi trabalhado com o conceito de acessibilidade espacial, que segundo a mesma autora (DISCHINGER et al., 2012), possibilita a compreensão da função e da organização do 


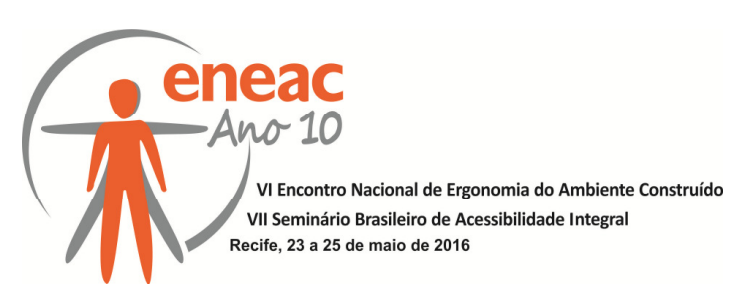

ambiente, bem como a participação do indivíduo nas atividades que o mesmo oferece, realizadas com segurança, conforto e independência. Para tal, são considerados somente os elementos construtivos que dificultam a realização das atividades de forma independente, definidos por Dischinger et al. (2012) como barreiras físico-espaciais.

Segundo Duarte e Cohen (2006), a acessibilidade ao espaço construído não deve ser relacionada apenas às pessoas com deficiência e sim, em proporcionar condições adequadas a acolher todos os usuários com igualdade e sem discriminação. Para que isso ocorra, é importante que estejam esclarecidos corretamente os conceitos de deficiência e restrição.

A Norma Brasileira de Acessibilidade define deficiência como sendo a "redução, limitação ou inexistência das condições de percepção das características do ambiente." (ABNT, 2004, p.3), ou seja, é a designação de um problema específico de uma disfunção no nível fisiológico do individuo. Sendo assim, vale ressaltar que a existência da mesma não implica necessariamente na incapacidade, uma vez que esta é resultado de um conjunto de fatores e não uma consequência somente das condições do indivíduo. Uma pessoa que necessita de cadeira de rodas para se locomover, por exemplo, é capaz de se deslocar com autonomia e segurança por uma calçada se os desníveis existentes puderem ser vencidos por rampas.

A partir deste dilema surge o conceito de restrição, que trata do grau de dificuldade gerado pelas características externas e as limitações do indivíduo ao se executar uma atividade (DISCHINGER, 2012). Este é o caso de uma criança que, mesmo não apresentando qualquer deficiência, pode sofrer restrição gerada pelo ambiente ao buscar um objeto em uma estante que esteja a uma altura fora do seu alcance.

\subsection{COMPONENTES DE ACESSIBILIDADE ESPACIAL}

Com o intuito de orientar as ações de avaliação e fiscalização dos edifícios públicos, Dischinger et al. (2012) classificaram os componentes de acessibilidade espacial em quatro categorias: comunicação, deslocamento, orientação e uso.

O componente comunicação refere-se às características que permitem a realização das atividades que envolvem a acessibilidade espacial a partir da troca de informações interpessoais ou pela utilização de equipamentos de tecnologia assistiva.

No componente deslocamento fazem parte os aspectos que proporcionam condições para que qualquer usuário se locomova de forma segura, confortável e independente pelos ambientes, tanto nos percursos horizontais quanto nos verticais.

Caracterizam-se como componentes de orientação espacial as condições que possibilitam o reconhecimento das funções atribuídas a cada ambiente ou equipamento, bem como a localização dos mesmos. Este componente auxilia na identificação dos locais onde se deseja chegar e o melhor percurso a ser utilizado.

O componente uso está relacionado às características apresentadas pelos ambientes e equipamentos que possibilitam a todos os usuários a realização das atividades desejadas com segurança, autonomia e conforto.

\section{METODOLOGIA}

Por se tratar de uma pesquisa qualitativa, ou seja, que a realidade não pode ser representada por variáveis, a influência das pessoas envolvidas sob a análise real da situação é significativa. Para reduzir tal interferência, optou-se pela realização de mais de 


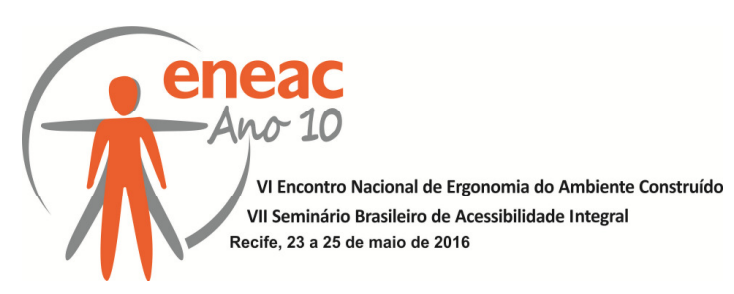

um método com o proposito de sanar possíveis limitações, de forma a se complementarem: visita exploratória e passeio acompanhado.

\subsection{VISITA EXPLORATÓRIA}

A visita exploratória consiste em registrar as condições espaciais do local e apresentar a visão do técnico sobre as mesmas. Para a realização deste método são utilizadas planilhas elaboradas com base em manuais do Conselho Regional de Engenharia e Agronomia (CREA) de diferentes estados brasileiros e em planilhas do Ministério Público do estado de Santa Catarina. Vale ressaltar, ainda, que este instrumento de coleta foi testado no trabalho já mencionado neste artigo.

Quadro 1 - Exemplo de parte da planilha aplicada no método visita exploratória

\begin{tabular}{|c|c|c|c|c|c|c|c|c|}
\hline \multirow{2}{*}{ № } & \multicolumn{2}{|c|}{ Legislação } & \multirow{2}{*}{ C. } & \multirow{2}{*}{ Itens a conferir } & \multicolumn{3}{|c|}{ Resposta } & \multirow{2}{*}{ Observações } \\
\hline & Lei & Artigo & & & Sim & Não & NA/I & \\
\hline \multicolumn{9}{|c|}{ CIRCULAÇÃO } \\
\hline 1.55 & $9050 / 04$ & 6.9.2.3 & E & $\begin{array}{l}\text { As maçanetas das portas são do } \\
\text { tipo alavanca? }\end{array}$ & & & & \\
\hline \multicolumn{9}{|c|}{$\begin{array}{lll}\text { Nota: } & \text { C. - Componente de acessibilidade espacial } & \text { NA/I - Não se aplica / Inexistente }\end{array}$} \\
\hline
\end{tabular}

Fonte: CANTARELLI (2014), adaptado de DISCHINGER et al (2012).

Cabe ressaltar que por proporcionar ao técnico um contato antecipado ao edifício, este método possibilita que o mesmo possa identificar trechos ou atividades mais críticas auxiliando na definição do percurso do método do passeio acompanhado.

\subsection{PASSEIO ACOMPANHADO}

O método baseia-se em acompanhar, sem conduzir ou ajudar, uma pessoa que sofra restrições na realização de atividades ao longo de uma rota pré-estabelecida. Durante o percurso o indivíduo deve verbalizar as facilidades e dificuldades enfrentadas. Para auxiliar na posterior análise dos resultados é interessante que haja além da gravação dos comentários realizados, o registro fotográfico relacionado aos momentos mencionados, assim como também. A amostra utilizada nesta pesquisa foram dois usuários: um com deficiência físico-motora (cadeirante) e outro com deficiência visual (cego).

\section{RESULTADOS E DISCUSSÃO}

A seguir são expostos e discutidos os problemas identificados no edifício analisado, conforme o componente de acessibilidade espacial ao qual se refere. Através dos quadros é possível distinguir ainda em qual dos métodos o problema foi detectado.

\section{Quadro 2 - Resultados referentes ao deslocamento}

\begin{tabular}{|c|c|c|c|c|c|}
\hline \multicolumn{2}{|c|}{ Problemas observados quanto ao deslocamento } & $\Rightarrow$ & Técnico & Cadeirante & Cego \\
\hline & \multicolumn{2}{|c|}{ Portas com vãos menores que $80 \mathrm{~cm}$ por folha } & & & \\
\hline & \multicolumn{2}{|c|}{$\begin{array}{l}\text { Portas com abertura em sentido inadequado nas } \\
\text { entradas do edifício }\end{array}$} & & & \\
\hline & \multicolumn{2}{|c|}{$\begin{array}{l}\text { Portas com abertura em sentido inadequado no } \\
\text { banheiro }\end{array}$} & & & \\
\hline
\end{tabular}




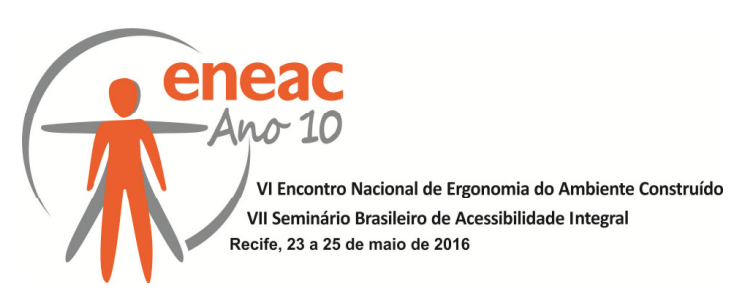

\begin{tabular}{|l|l|l|l|}
\hline & Catraca no acesso da biblioteca & & \\
\cline { 2 - 4 } & $\begin{array}{l}\text { Corredores estreitos entre as estantes da } \\
\text { biblioteca }\end{array}$ & & \\
\hline $\begin{array}{l}\text { Corredores sem espaço para rotação de } 180^{\circ} \text { de } \\
\text { uma cadeira de rodas entre as estantes da } \\
\text { biblioteca }\end{array}$ & & & \\
\hline
\end{tabular}

Fonte: CANTARELLI, 2014.

Com relação ao componente de deslocamento o ponto de vista técnico foi o que mais indicou problemas na edificação. No método do passeio acompanhado o único usuário que enfrentou problemas foi o cadeirante. Vale ressaltar ainda, que o indivíduo foi impossibilitado de realizar uma das atividades previstas no percurso - acessar a biblioteca - em função da existência de uma catraca na entrada da mesma, como pode ser observado na imagem apresentada no Quadro 2.

Quadro 3 - Resultados referentes ao uso

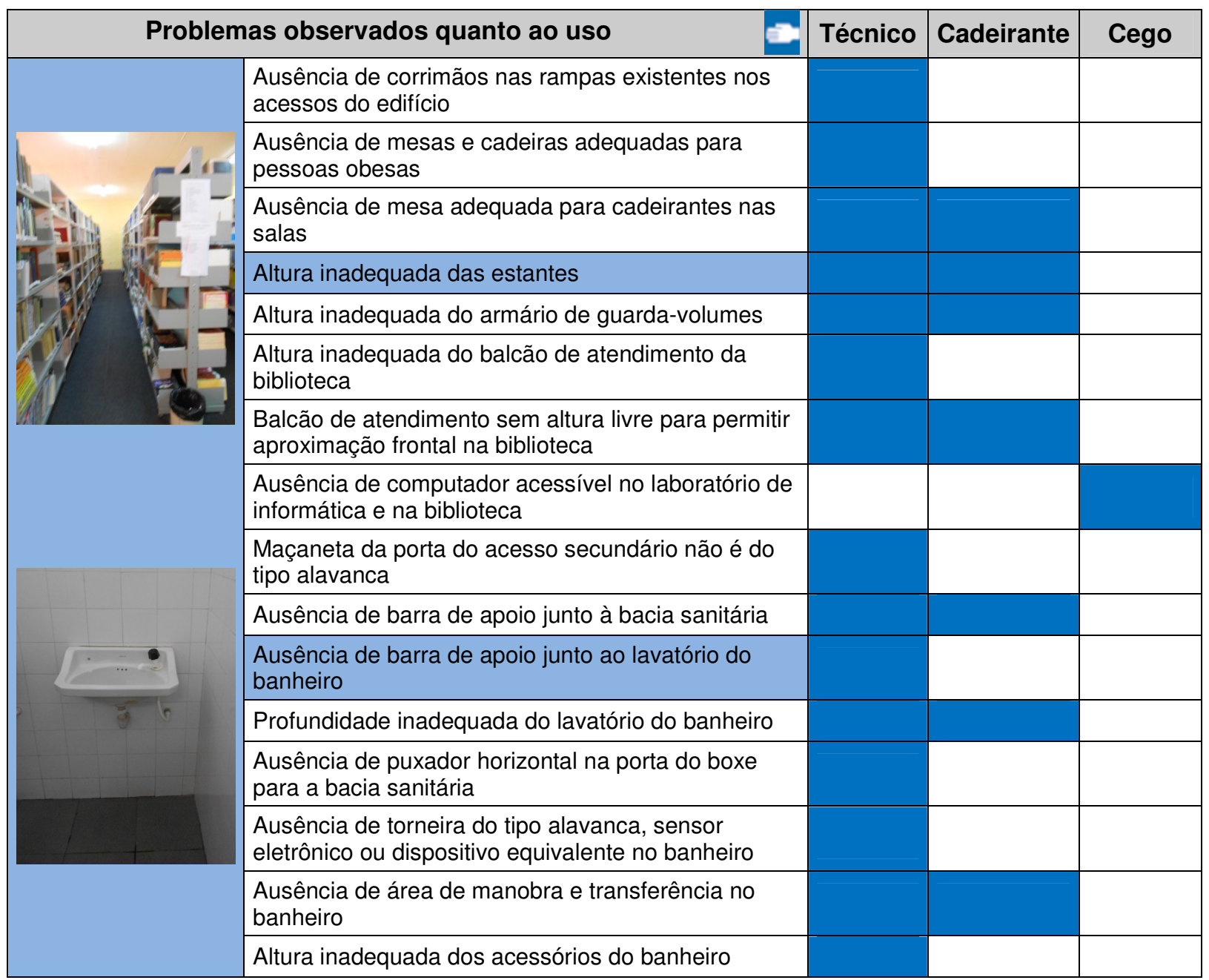

Fonte: CANTARELLI, 2014.

Quanto ao componente uso o ponto de vista técnico foi novamente o que detectou o maior número de problemas. Contudo, grande parte destes itens também foi apontada como dificuldade pelo usuário em cadeira de roda no passeio acompanhado. É importante salientar que, apesar do individuo com deficiência visual ter apontado apenas uma 


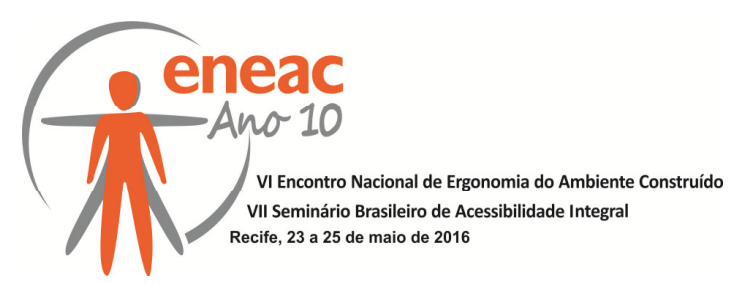

dificuldade, conforme apresentado no Quadro 3, a mesma o impossibilitou de realizar uma das atividades previstas no roteiro do passeio acompanhado (utilizar os computadores, tanto da biblioteca, quanto do laboratório de informática).

Quadro 4 - Resultados referentes à comunicação

\begin{tabular}{|l|l|l|l|l|}
\hline \multicolumn{2}{|c|}{ Problemas observados quanto à comunicação } & Técnico & Cadeirante & Cego \\
\hline & $\begin{array}{l}\text { Ausência de sistema de alarme de incêndio } \\
\text { simultaneamente sonoro e luminoso no interior do } \\
\text { edifício }\end{array}$ & & & \\
\hline & $\begin{array}{l}\text { Ausência equipamento de tecnologia assistiva } \\
\text { (terminal de computador) que permita a } \\
\text { comunicação do surdo e/ou mudo com os } \\
\text { funcionários na biblioteca }\end{array}$ & & \\
\hline & $\begin{array}{l}\text { Ausência de sinalização de emergência junto a } \\
\text { pelo menos uma bacia sanitária nos banheiros }\end{array}$ & & \\
\hline
\end{tabular}

Fonte: CANTARELLI, 2014.

Como pode ser observado no Quadro 4, os problemas relacionados ao componente de comunicação foram identificados apenas pelo método da visita exploratória. Contudo, é importante ressaltar que os usuários que realizaram os passeios acompanhados não tinham restrição na fala e/ou na audição, o que justifica os mesmos não terem tido dificuldades com a ausência destas características.

\section{Quadro 5 - Resultados referentes à orientação espacial}

\begin{tabular}{|l|l|l|l|l|}
\hline Problemas observados quanto à orientação espacial ? & Técnico & Cadeirante & Cego \\
\hline & Ausência de informação visual no edifício & & & \\
\hline & Ausência de suporte tátil no edifício & & & \\
\hline & $\begin{array}{l}\text { Ausência de iluminação de emergência junto à } \\
\text { rampa }\end{array}$ & & & \\
\cline { 2 - 6 } & Ausência de indicação das saídas de emergência & & & \\
\cline { 2 - 6 } & Ausência de identificação dos objetos do banheiro & & & \\
\hline
\end{tabular}

Fonte: CANTARELLI, 2014.

A visita técnica foi o método pelo qual se identificou maiores problemas no que diz respeito ao componente orientação espacial. No entanto, os dois usuários que realizaram o passeio enfrentaram dificuldades frente à ausência de informações no edifício. Cabe salientar que, por tratarem de situações de risco os itens de sinalização de emergência não foram avaliados por este método.

\section{INTERVENÇÃO}

Com base nos resultados obtidos, foram avaliadas possíveis soluções para cada um dos problemas encontrados e, a partir disto, elaborada proposta de intervenção, na qual foram detalhadas e localizadas na planta baixa do edifício as recomendações sugeridas. Parte 


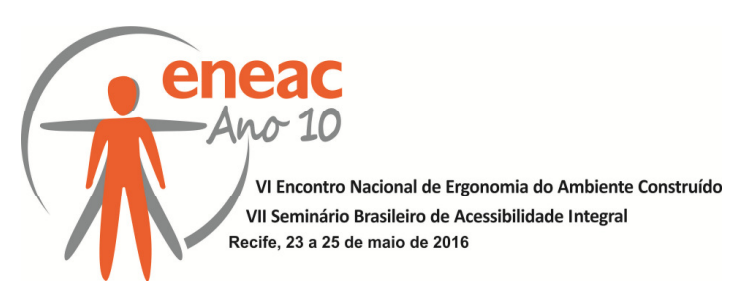

deste estudo pode ser observada nos quadros a seguir onde são expostas as intervenções recomendadas para os acessos do edifício e para os sanitários.

\section{Quadro 6 - Intervenções recomendadas no acesso ao prédio}

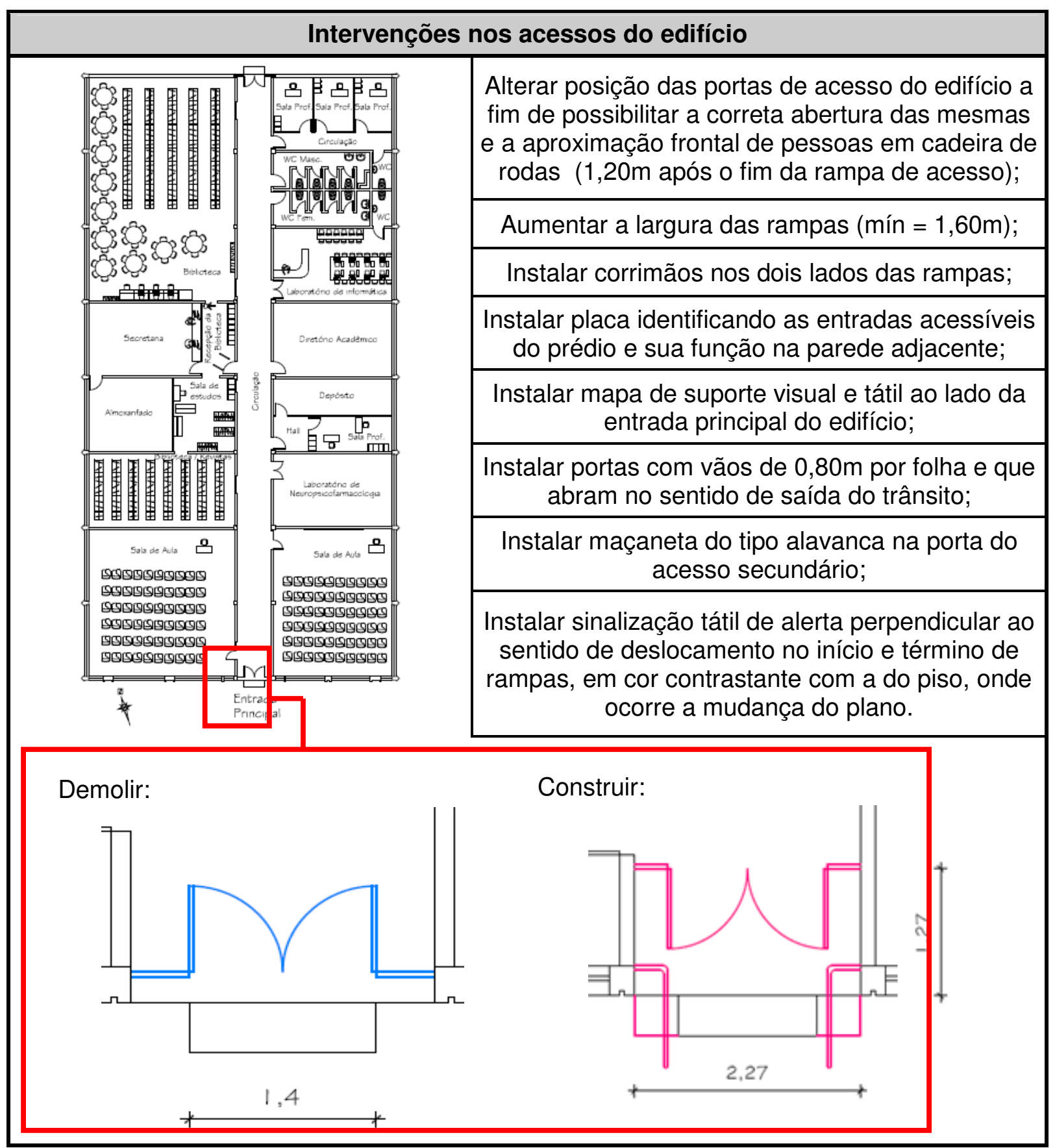

Fonte: CANTARELLI (2014) 


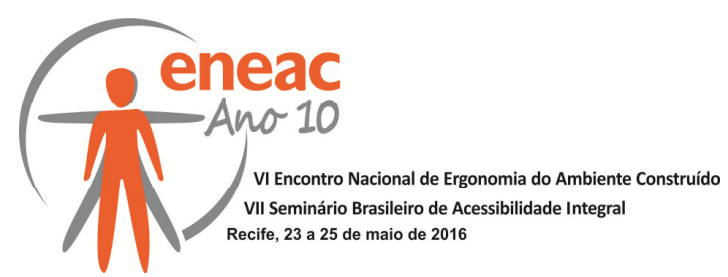

Quadro 7 - Intervenções recomendadas nos sanitários

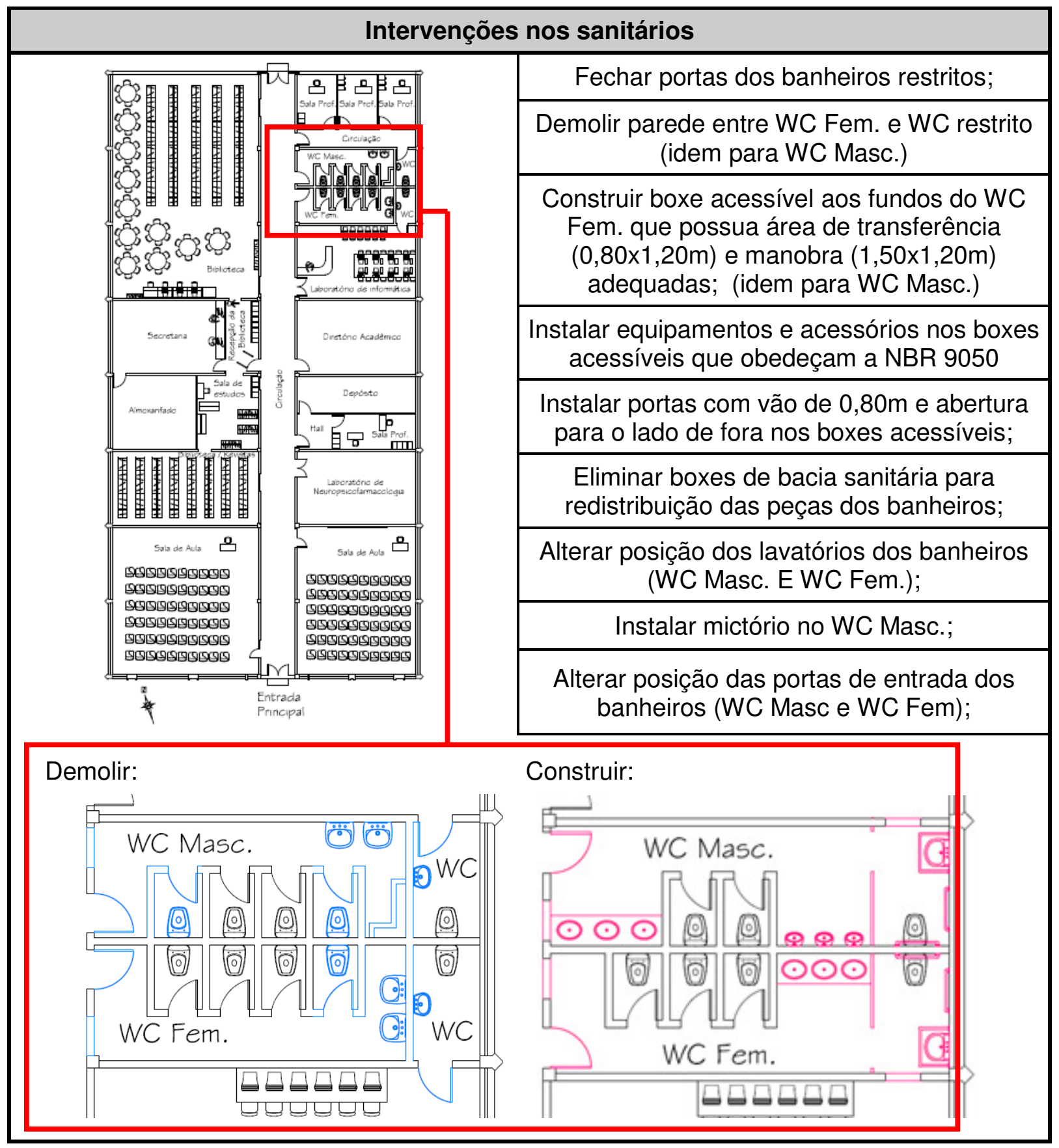

Fonte: CANTARELLI (2014)

\section{CONSIDERAÇÕES FINAIS}

Analisando os resultados deste trabalho é possível observar a relação entre as limitações dos indivíduos e os quatro componentes de acessibilidade espacial. Sendo assim, é imprescindível que todos eles sejam atendidos em sua totalidade para que seja possível proporcionar ambientes que ofereçam conforto e segurança a todos os usuários, independente de suas habilidades e restrições. Para garantir tal situação é necessário mais que a aplicação dos critérios e parâmetros recomendados pelas normas. É preciso, ainda, 


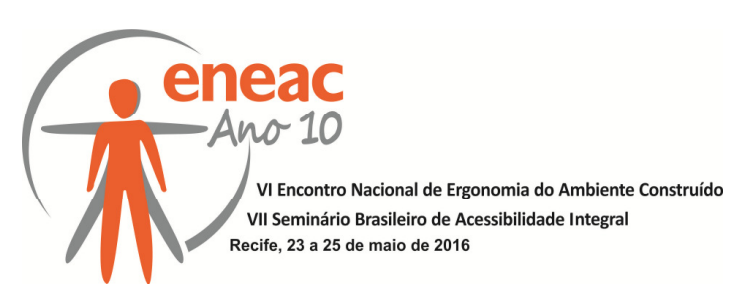

ter conhecimento das restrições advindas das diferentes deficiências para, então, distinguir corretamente os obstáculos existentes e definir as melhores soluções para os mesmos.

Sendo assim, entende-se que a aplicação do método de passeio acompanhado foi de grande relevância neste trabalho, uma vez que aproximou o técnico de situações concretas vivenciadas por pessoas com deficiência em seu dia a dia. Considera-se importante, também, a utilização da visita exploratória, que além de auxiliar na comparação das características apresentadas no edifício e nas especificações existentes na norma, possibilita o reconhecimento prévio do edifício, facilitando a elaboração de percursos que envolvam situações mais problemáticas para a execução dos passeios. Vale ressaltar, ainda, que a união dos dois métodos adotados proporcionou que fossem sanadas as possíveis limitações de cada um, de forma a se complementarem.

Esta pesquisa teve como impasse principal o envolvimento de pessoas como instrumento de identificação dos problemas a partir do passeio acompanhado. Por depender de outros indivíduos foram enfrentadas dificuldades na definição dos horários de realização dos passeios, assim como no deslocamento - principalmente no caso da pessoa em cadeira de rodas.

Por fim, espera-se que as propostas de intervenções elaboradas para o edifício do Departamento de Fisiologia e Farmacologia I da UFPel, objetivo principal deste trabalho, possam, além de contribuir com a adaptação da UFPel às condições de acessibilidade espacial exigidas na legislação brasileira, servir de referência para futuros projetos e despertar o interesse na comunidade escolar, salientando a importância do tema em questão.

\section{REFERÊNCIAS BIBLIOGRÁFICAS}

ASSOCIAÇÃO BRASILEIRA DE NORMAS TÉCNICAS (ABNT). NBR-9050: Acessibilidade a edificações, mobiliário, espaços e equipamentos urbanos.Rio de Janeiro, 2005.

BRASIL. Constituição da República Federativa do Brasil de 1988.

BRASIL. Portaria no 3.284, de novembro de 2003. Dispõe sobre requisitos de acessibilidade de pessoas portadoras de deficiências, para instruir os processos de autorização e de reconhecimento de cursos, e de credenciamento de instituições.

CANTARELLI, Rafaela Mezomo. Acessibilidade espacial na UFPel: Estudos de casos e proposta de intervenção para um prédio com finalidade acadêmica. Trabalho de Graduação - Curso de Engenharia Civil - Universidade Federal de Pelotas. Pelotas, 2014.

CONSELHO REGIONAL DE ENGENHARIA E AGRONOMIA DO RIO GRANDE DO SUL (CREA-RS). Roteiro de visita: Acessibilidade a edificações, mobiliário, espaços e equipamentos urbanos. Porto Alegre, 2007.

CONSELHO REGIONAL DE ENGENHARIA E AGRONOMIA DO PARANÁ (CREA-PR). Acessibilidade: Responsabilidade Profissional. 4.ed. Curitiba, 2011.

DISCHINGER, Marta; ELY, Vera Helena Moro Bins; PIARDI, Sonia Maria Demeda Groisman.

Promovendo acessibilidade espacial nos edifícios públicos: Programa de Acessibilidade às Pessoas com Deficiência ou Mobilidade Reduzida nas Edificações de Uso Público. Florianópolis: MPSC, 2012. 161 p.: il., tabs., mapas.

DISCHINGER, Marta. Acessibilidade e Desenho Universal. 2005. Notas de aula.

DUARTE, Cristiane Rose de Siqueira; COHEN, Regina. Proposta de Metodologia de Avaliação da Acessibilidade aos Espaços de Ensino Fundamental. In: Anais NUTAU 2006: Demandas Sociais, Inovações Tecnológicas e a Cidade. São Paulo: USP, 2006. 